\title{
When Liquid Droplets Take a Turn
}

\section{A new model reveals that patterns of internal fluid flow control whether self-propelling water droplets in oil follow linear or curved trajectories.}

\section{By Sophia Chen}

$\square$ luid droplets that propel themselves through liquid environments could have biomedical applications-for example, as microrobots that deliver cargoes of medicine. While researchers have created self-propelling droplets driven by surface tension, they still don't understand the mechanisms that control the droplets' direction and speed. Now, Masatoshi Ichikawa of Kyoto University in Japan and colleagues have analyzed the conditions that cause self-propelling droplets to take linear or curved trajectories [1].

The team studied water droplets between 60 and $800 \mu \mathrm{m}$ across as they moved through oil that contained a surfactant. The droplets moved as a result of the Marangoni effect, in which an unequal distribution of surfactant molecules on the surface of each droplet creates a surface-tension gradient. The researchers found that larger droplets tended to follow more tightly curved paths than smaller droplets.

To understand the cause of this difference, Ichikawa and

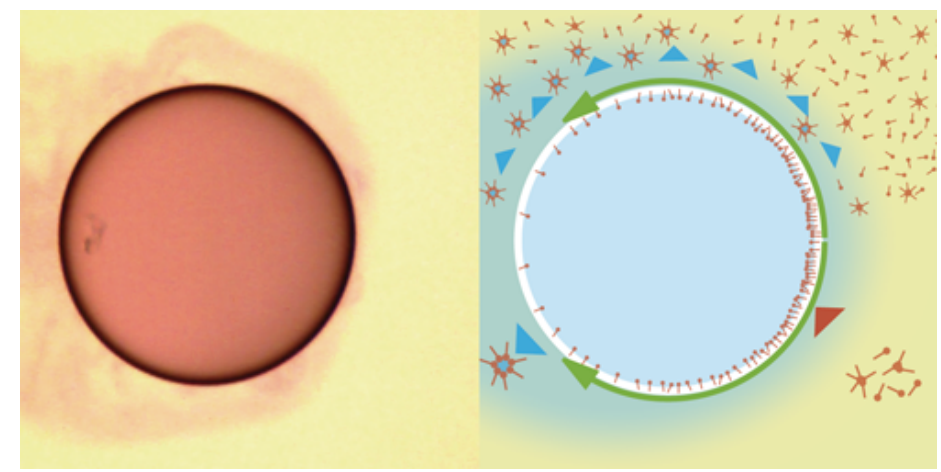

Credit: S. Suda et al. [1] colleagues created a 3D model describing the concentration of surfactant on the surface of the droplets. They also studied the droplets' internal flow, by observing the paths of small tracer particles. They characterized this flow as the sum of multiple patterns of fluid motion present in each droplet, including radial, dipolar, and quadrupolar motion. These patterns of motion were determined by the surface-tension gradients created by the uneven surfactant distribution on each droplet. In turn, such patterns controlled how the droplets moved. In particular, the team found that the angular difference between the dipolar and quadrupolar flows within droplets was strongly correlated with more curved droplet trajectories. In larger droplets, this angle changed more easily, causing the tightly curved trajectories. The researchers say that this fundamental mechanism may also influence the swimming behavior of amoebas.

Sophia Chen is a freelance science writer based in Columbus, Ohio.

\section{REFERENCES}

1. S. Suda et al., "Straight-to-curvilinear motion transition of a swimming droplet caused by the susceptibility to fluctuations," Phys. Rev. Lett. 127, 088005 (2021). 